\title{
Blockchain Technology for Global Decentralized Manufacturing: Challenges and Solutions for Supply Chain in Fourth Industrial Revolution
}

\author{
Babak Zareiyan $^{1^{*}}$ and Mehdi Korjani ${ }^{2}$ \\ ${ }^{1} \mathrm{PhD}$ in Civil Engineering from University of Southern California, USA \\ ${ }^{2} \mathrm{PhD}$ in Electrical Engineering from University of Southern California, USA
}

Received: May 02, 2018; Accepted: May 21, 2018; Published: May 31, 2018

*Corresponding author: Babak Zareiyan, PhD, Department of Civil Engineering, University of Southern California, USA, E-mail: zareiyan@usc. $e d u$

\begin{abstract}
In this paper we introduce 3D-Chain, a decentralized solution based on blockchain technology to global manufacturing challenges for industry 4.0 revolution [1]. 3D-Chain aims to provide an ecosystem for manufacturers, designers, and consumer to interact efficiently without any restriction. Within this framework, the benefits of manufacturing become a global common infrastructure for all. Moreover, demand for personalized design is exponentially growing every year and soon dominate the manufacturing and the economy. However, manufacturing new items and personalized them are very expensive and time-consuming. 3D-Chain facilitates the mass customization through its network to answer this global demand and it is designed to incentivize and reward beneficial players. Developing and building innovation processes that prioritize global growth in economy, supporting software infrastructure for sustainable merge of fabrication technologies into a new decentralized manufacturing market, addressing cross-functional components in manufacturing and design, decentralizing manufacturing to increase performance and reduce waiting time and cost, unlocking mass customization, and Evaluating consumers, business, manufacturers, services providers, and suppliers are 3D-Chain's mission and objectives.
\end{abstract}

Keywords: 3D-Chain; Blockchain; Decentralize manufacturing; Additive manufacturing; Automated fabrication technologies;

\section{Introduction}

\section{The future of manufacturing}

Manufacturing is a critical contributor to productivity, innovation, and trade. Time of big growth always has been supported by manufacturing revolutions (Steam engine, mass production, and automation). Today, rates of economic growth in major economic blocks are declining and the global manufacturing is facing a fierce competition over every scrap of market share. Many opportunities in the industrial value creation in the early industrialized countries are shaped by the development towards the fourth stage of industrialization, the so-called Industry 4.0 [2]. Industry 4.0 is the collection of cutting-edge technologies that support effective and accurate engineering decision-making in real time through the introduction of various technologies and the convergence with the existing manufacturing technologies [3]. There are different technology trends that are the building blocks of Industry 4.0 [4-6]. Figure 1 shows important factors in global manufacturing within Industry 4.0 revolution. In this paper we focus on solutions to overcome current challenges in Industry 4.0 .

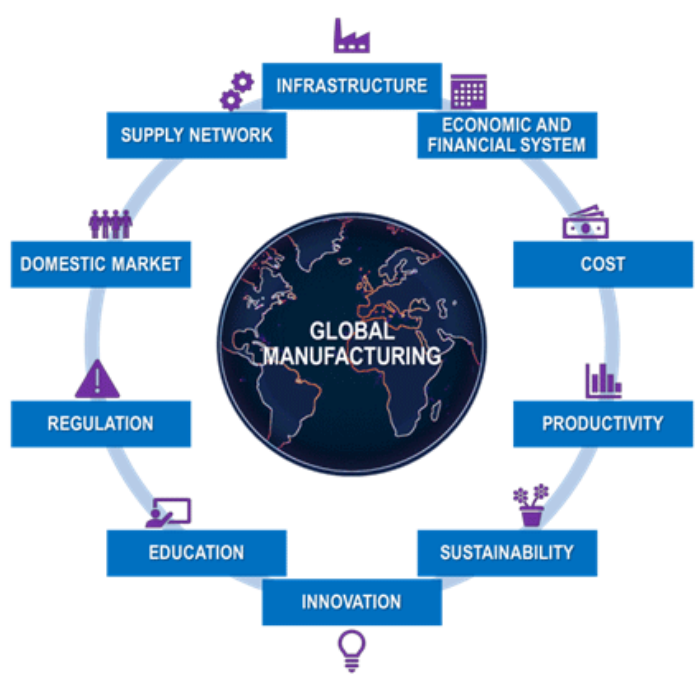

Figure 1: Important factors in global manufacturing competitiveness

Potential technical and economic benefits for manufacturers and production equipment suppliers using industry 4.0 are presented in [4,7]. Studies show that decentralization and network building change the manufacturing landscape from an Industry 4.0 perspective [8]. The increasing utilization of information and communication technology allows digital engineering of products and production processes alike. Modular simulation and modeling techniques allow decentralized units to flexibly alter products and thereby enable rapid product innovation. In this 
transformation, sensors, machines, workpieces, and IT systems will be connected along the value chain beyond a single enterprise. These connected systems can interact with one another using standard Internet-based protocols and analyze data to predict failure, configure themselves, and adapt to changes [4].

\section{D Printing}

A compelling solution to manufacturing growth is Additive Manufacturing (AM) also known as 3D Printing which can expedite the whole manufacturing process while decreasing cost and hassle of making new products [9]. 3D Printing creates physical objects from a digital file using layer upon layer printing approach [10].

For manufacturers, 3D Printing is not the future, it is a production method like any other. A survey shows manufacturers consider additive manufacturing a vital part of their business operation and recognize an urgent need to increase their investment into innovation and R\&D [11]. $60 \%$ of manufacturers already use 3D printing in some way, and $30 \%$ plan to adopt the technology in the future (Figure 2). However, the full potential of 3D Printing is far from tapped.

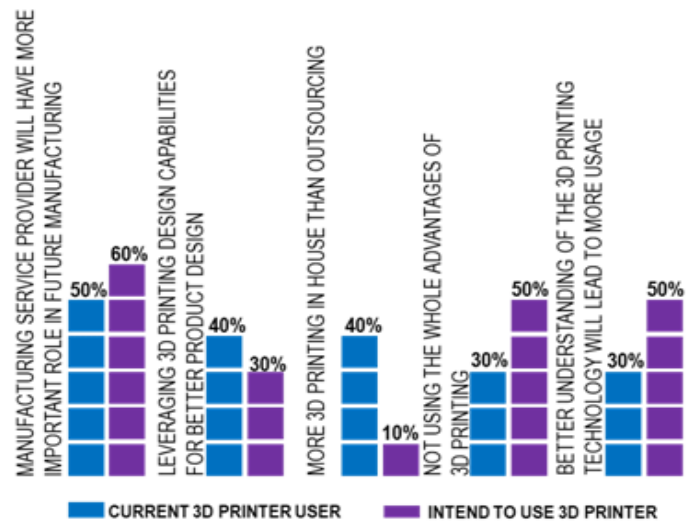

Figure 2: Current manufacture user of 3D Printing technology vs. future users; Source data [12]

The 3D Printing technology has roots that go back for decades. But for years, 3D Printing did not apply in the commercial manufacturing. Nevertheless, 3D Printing presents compelling business opportunities and we are now observing different application of 3D Printing due to decrease in cost, technological advances, and flexibility in design.

Prototyping and proof of concept are the leading use of 3D printing (Figure 3). However, 3D Printing market is maturing, and it is evolving from its roots in the volunteer maker movement into a highly competitive business. This new technology can reduce the cost and barriers in manufacturing. There are reasons to believe that 3D Printing will begin to infiltrate the mainstream, with success belonging to products and services that focus on the object being made, rather than the hobbyist's thrill in the futuristic manner of how it was made.

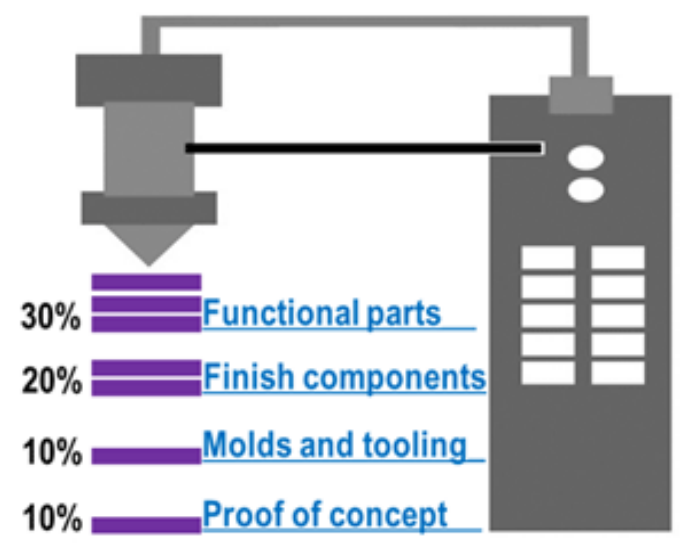

Figure 3: Current top user of 3D Printing technology; source data: [13]

- The primary market (3D Printing systems, materials, supplies, and service) will grow $30 \%$ each year.

- Increase applications of 3D printing in medical and dental industries are driven by capability of new generation of 3D Printers to fabricate precise complex geometries [14].

- While US is the leader in additive manufacturing applications followed by Europe, there is a clear trend in the adoption of additive manufacturing by Asian countries. (Figure 4)

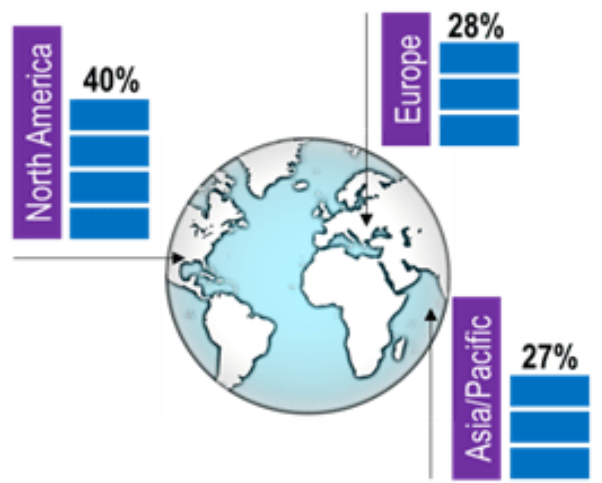

Figure 4: Global 3D Printing market by region; Source data [13]

3D Printing allows individuals to design and produce objects on demand. The most exciting part of this technology is beyond productivity and extends to smarter, scalable, and better products. It will create a microeconomic shift and relocate the process to home market. Because of this movement:

- The final product will be closer to consumer market

- Factories will be smaller and decentralized

- Scalability and flexibility are important not mass production

- A new area of globalization is forming 
Another advantage of utilization of industry 4.0 is customization of mass-produced parts by combining injection molding and additive manufacturing [15]. Various techniques are presented that aid the design process of individualized products and organization of their production in the context of realization of the mass customization strategy, which allows a shortened time of development for a new product $[3,16,17]$.

\section{Problem}

\section{Issues in current 3D printing industry}

Mainstream application of 3D Printing requires investment in tools and materials for any specific product. You need to have skills to design your idea, have right tools to develop a prototype, and be familiar with all the optimization tools that are needed to turn a 3D file into a physical object. Having luxury of enough time, enough money and right skills to go through the whole process is very rare. The main restrictions are:

\section{Size restriction}

Future growth in large scale 3D printing requires investing a lot of money or using external 3D printing services.

\section{Material restriction}

To 3D Print a product, specific material is necessary. Plastic is the most common 3D printing material followed by resin. Knowing all the materials, analyzing it, and finding/purchasing a new 3D Printer for that specific material are very challenging.

\section{D printing technology}

There are several 3D printing technologies, each has different way to process input material and create a final product. Consumer must learn all possible technologies and pick most appropriate one for their applications. Therefore, finding a manufacturing company to make the design compatible with required specifications demands lots of time and efforts.

\section{Innovation and intellectual property issues}

The shift in global demand for 3D Printed good requires very different service with different features and price points. Offering a variety of designs while protecting copyright is challenging for designers and manufacturers. These questions arise in global 3D Printing and design environment which is different from past with more complexity, uncertainty, and risk.

\section{Challenges in current supply chain of manufacturing}

With manufacturing focusing on adopting new technology, ensuring the supply chain is ready for growth is a priority. Currently, the commercial adoption of 3D Printing technology has been delayed by insufficient infrastructure.

Today, manufacturing is not about where to locate production, but it is about changing in customer's demand, resiliency in supply chain, and cost factors. A new decentralized supply chain is required to serve application of 3D Printing and automated fabrication technologies in global manufacturing industry as the source of consumer as well as the source of low-cost production. A platform that is organized and operates in fundamentally different way to create a collaboration around the world of design, fabrication, marketing, and service in an intelligent and secure environment. (Figure 5)

\section{Inventory cost}

\section{Flexibility in design}

Supply chain challenges in manufacturing

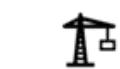

Mining

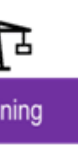

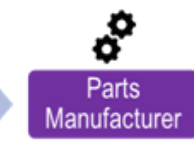
.
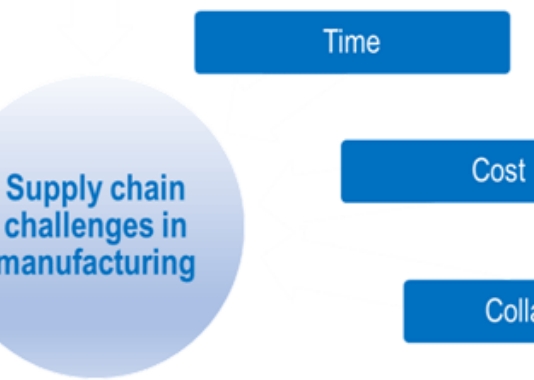

\section{ost}

Collaboration

Figure 5: Challenges in current supply chain of manufacturing

\section{D-Chain: A Decentralized Network for Future Manufacturing}

Despite significant growth, there is a wealth of untapped potential in 3D Printing and automated fabrication technologies. What if we combine existing manufacturing process and innovation in technology sector to create next manufacturing invention, a productive environment that does complex non- 


\section{repetitive tasks?}

Blockchain technology gives manufacturing an opportunity to transform the supply chain into a decentralized environment to achieve a new level of productivity and efficiency. The only thing missing is a platform which enables secure and sustainable transactions.

We need a platform focuses on addressing the impact of digitization on manufacturing strategies and key shortcomings of current infrastructure on adaptation of 3D Printing and automated fabrication technologies (Figure 6). It needs to transform hardware-based supply chain in manufacturing to one that is software-defined to prevent unexpected failure in supply chain as one of the greatest risk. It also needs to improve the visibility and flexibility of supply chain, reduces the risk of partnering with new designer, supplier, and producer, and creates a new value chain.

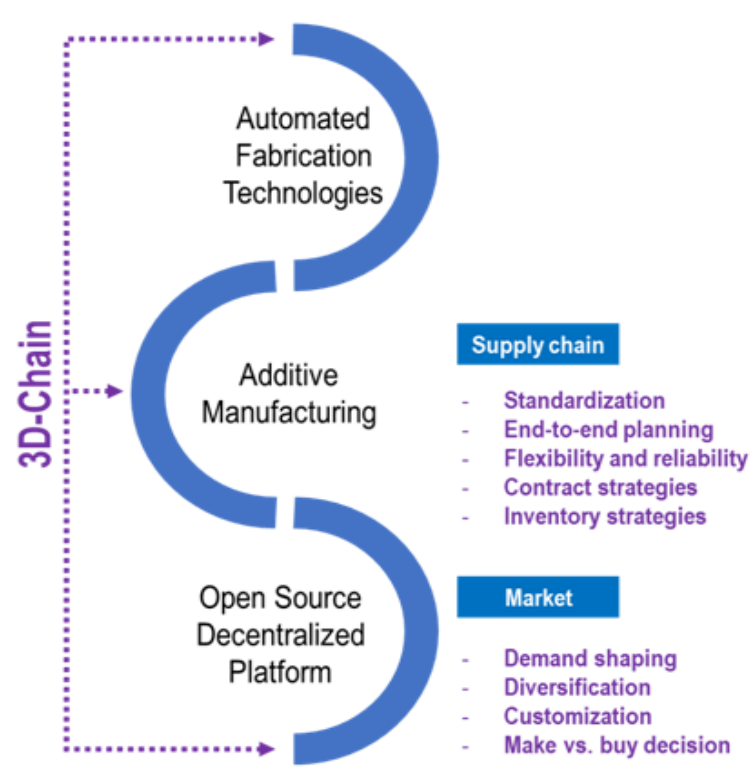

Figure 6: 3D-Chain: a decentralized platform for automated fabrication technologies and additive manufacturing

We presented in our previous paper 3D-Chain platform for manufacturing as a secure platform uses greater intelligence in product design and manufacturing to boost resource efficiency and track activity by implementing blockchain technology [1]. 3D-Chain introduces the first supply chain using cryptography for expansion of 3D Printing and automated fabrication technologies to consumer market (B2C) and industrial applications (B2B). 3D-Chain is a fully integrated system across the entire manufacturing industry, from the moment a design is developed by designer to the final delivery to consumer. It helps forwardthinking players to take advantage of decentralized technology for next generation of manufacturing.

3D-Chain is highly focused on providing new growth opportunity for manufacturing market. Our goals are, increasing adaptation range of new technologies, saving cost, reducing the risk of faulty design, protecting copyright, and expanding customized design and fabrication using advantages of blockchain technology.

Many designer and engineers are outsourcing their 3D printing demands as speed and precision of external 3D printing providers has increased dramatically in last years. 3D-Chain will be market place to guarantee direct communication between parties, production, and delivery. 3D-Chain is a network of 3D Printers in different sizes for scale variation in manufacturing, a decentralize network which provides a platform of material for 3D Printing. Moreover, consumer, designers, and manufacturer can connect to the 3D-Chain network and select a specific technology for their applications.

3D-Chain is a new, creative, and collaborative environment that provides a platform for those with ambitions to expand into new markets, companies or individuals that are looking to bring more value to their consumer, innovator, researcher, and scholar in manufacturing industry. It also sets a decentralize third party governance program to lower the risk of uncertainty and ensure the compliance with contractual obligations. With 3D-Chain, the meaning of each cryptographic transition is only accessible for interpretation by the parties involved. The contract is completed and stored on the main blockchain which gives designers more visibility and allows contributors direct communication.

3D-Chain provides an environment to fully embrace the potential of 3D Printing and automated fabrication technologies in all steps of products' lifetime (Figure 7). This will increase the performance and expand the application to wider range of industries. Increase in return of investment is another result of decentralize platform and gain a competitive advantage over other manufacturers.

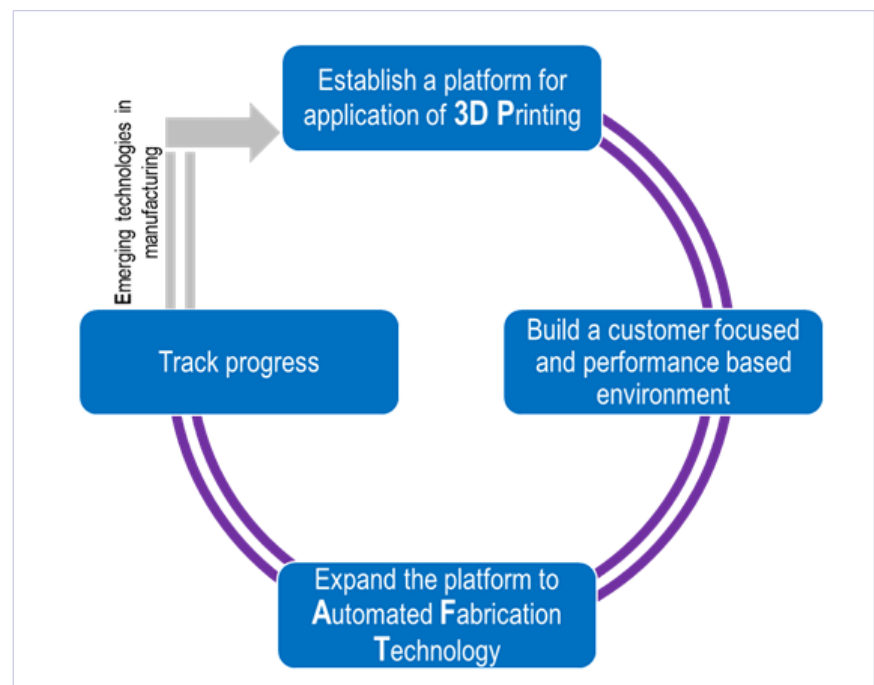

Figure 7: Development of the 3D-Chain's network

\section{Benefits of using decentralized 3D printing technology}

3D-Chain provides a competitive market to overcome the restrictions in mainstream applications of 3D Printing and automated fabrication technologies.

Manufacturing industry can benefit from 3D-chain by: 
- Providing a platform for people who are creative and explorer, those who want to change the world of design while protecting the copyright.

- Establishing a virtual marketplace for making, buying and selling products. Anyone with a 3D Printing machine or fabrication technology can provide a manufacturing service (open a shop, make a brand, promote it, share it).

- Helping people who are not familiar with the design tools to create objects.

- Allowing customers to adjust a few parameters and giving them enough control to take ownership of a product without overwhelming them.

- Facilitating fast and affordable fabrication of prototypes to set ideas in motion.

- Bringing group of designers and 3D Printers together to share ideas and to make things which they are impossible or very expensive to make in existing manufacturing ways.

- Creating small business around design and manufacturing instead of large scale factories.

- Sharing products, technologies and ideas in a secure environment.

- Expanding the design/ manufacturing into as many hands as possible.

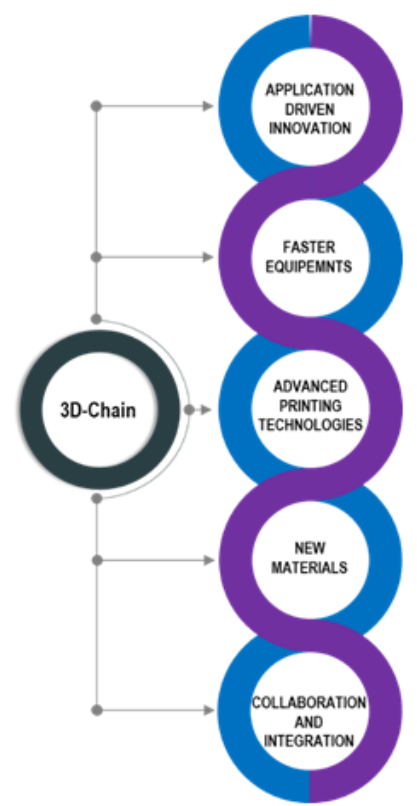

Figure 8: 3D Printing future trend will be available through 3D-Chain for users

- Customizing and personalizing items for individuals (Figure 9). Eventually, using recyclable or sustainable materials which preserve natural resources.

- Eliminating mass production and managing the inventory through real-time production.
The future 3D printing industry will be more and more application-driven (Figure 8) to meet specific demands for business and consumer. By connecting network of 3D Printers and fabrication technologies with different capabilities on 3D-Chain platform, manufacturers and consumer have the freedom to focus on the application which will cause the real transformation in the industry. Collaboration is also the key for creating a sustainable supply chain for future manufacturing. 3D-Chain increase collaboration between existing and future players in its decentralized platform, an ecosystem which integrates different parts of 3D Printing industry and automated fabrication technology (hardware, software, service, ...) to drive innovation and serve consumer. This results in a leaner supply chain and fewer risks.

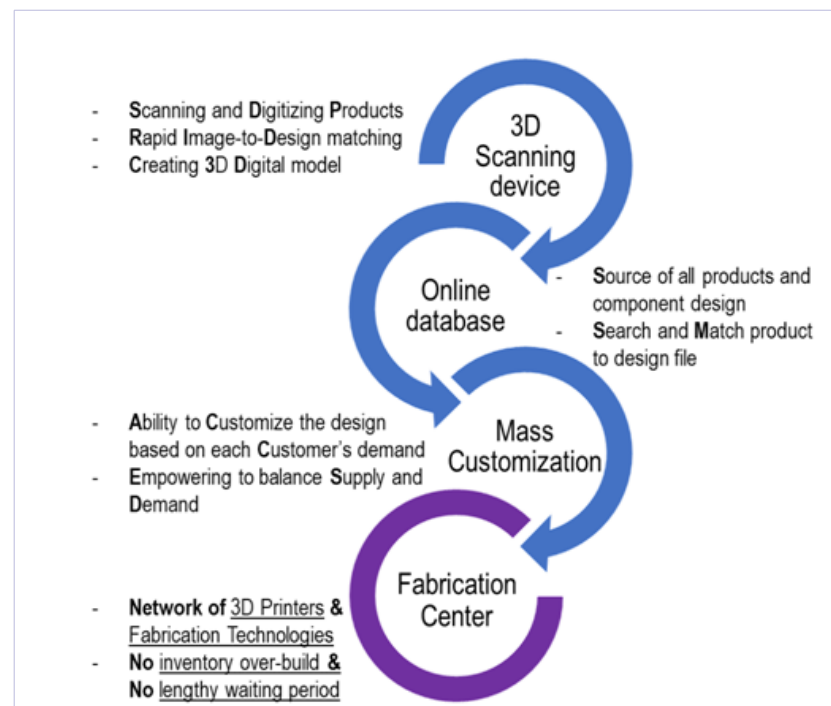

Figure 9: 3D-Chain unlocks area of mass customization in a decentralized and secured environment

\section{D-Chain's ecosystem and marketplace}

The challenges that come with additive manufacturing in terms of production and decision making are numerous. Meeting these challenges drives us here at 3D-Chain to continue to create a new platform for future growth in production.

3D-chain can turn data into objects and objects into data on demand in a protected and secure environment. This approach facilitates the productivity in manufacturing, increase efficiency, and lead to the global growth and distribution of wealth as our goal.

The ecosystem is a global decentralized platform where consumers can buy designs directly from designer, personalize a design, select among different materials and specifications, optimized the design for a specific fabrication technology, and send the design to appropriate manufacture and receive it in manageable time.

Raw material supplier, component supplier, 3D Printing machine manufacturer, fabrication technology provider, researcher, service provider, distributer, and end user define the ecosystem. The ecosystem's users (Figure 10) are providers of: 


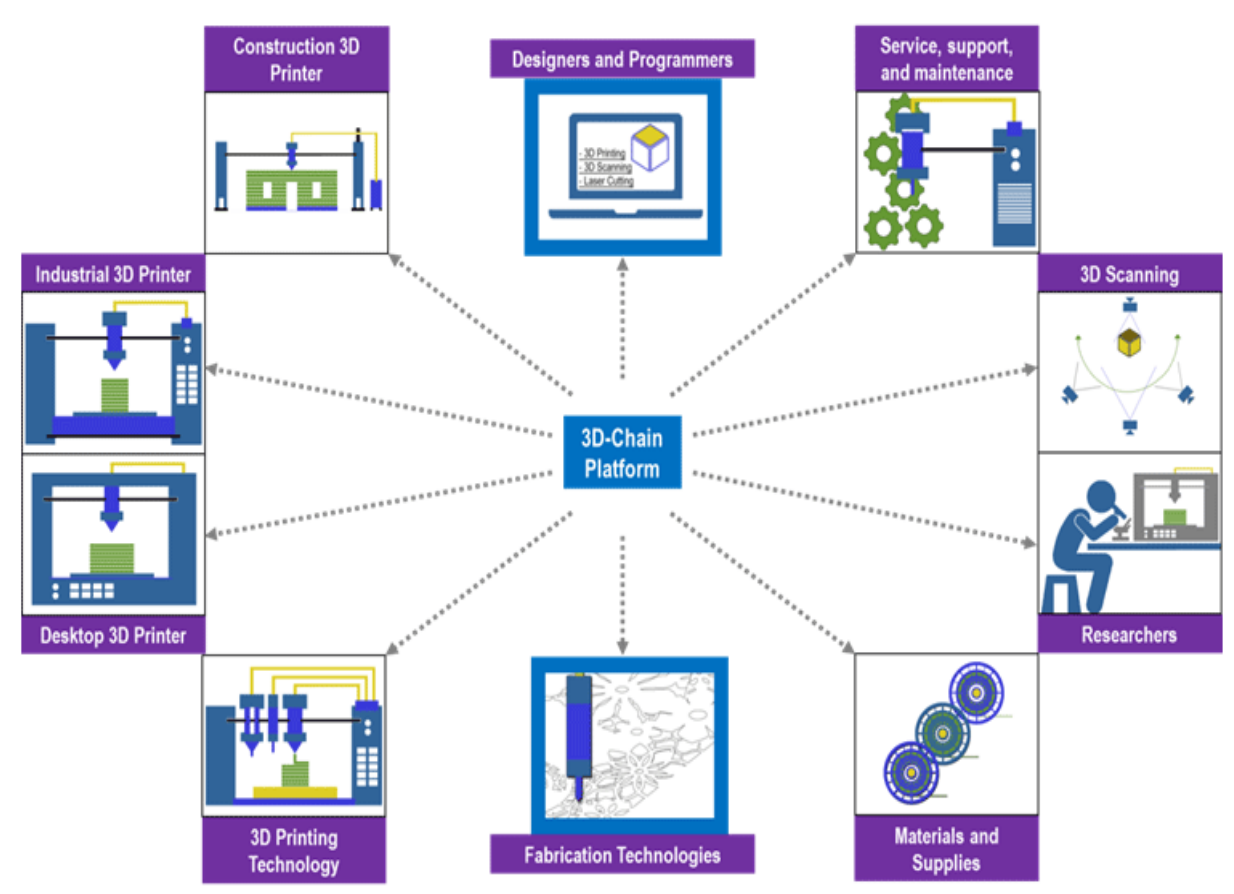

Figure 10: 3D-Chain's ecosystem

- 3D Printing solutions (Desktop, Industrial)

- 3D Printing services

- 3D Printing materials (Plastic, Metal, Concrete, and Other Materials)

- 3D Printing accessories

- 3D Printing consultation

- 3D Printing software (Printing, Design, and Inspection)

- 3D Scanning services

- Manufacturers, fabrication technology providers, and start-up companies

- Designers and Computer-aided design (CAD) developers

- Distributers and wholesalers

- End users and consumer

- Educators and researchers in 3D Printing and automated fabrication technology

Incentive of consumer to use 3D-Chain platform is to access transparent prices for a wide range of designs, designers, and manufacturers. 3D Printing and fabrication technology providers compete for consumers/designers and interact with them directly with fraction of cost of traditional systems. In addition of accessing the network, consumer and designers can send a design or an idea with great confidentiality inherent from the network and make sure that their work is protected with copyright.

\section{D-Chain's network based on Blockchain}

Blockchain is a decentralized technology, aiming to solve trust issue between users. Bitcoin is the most popular example of Blockchain technology. It is a digital currency where miners validate all transactions. It uses a distributed ledger that is updated by consensus among the community, rather than held privately.

3D-Chain's network uses blockchain technology to facilitate communication and distribute resources in the network. This technology provides a scalable, decentralized, and selforganizing network that ensures sustainability in the platform and reliable communication between consumers, designers, and manufactures. As such, it can be built on any open, decentralized framework that satisfies certain requirements. The platform requires:

- Global Network: The platform opens with 3D Printing technology and develops to an entire world of manufacturers, designers, and consumers.

- A Scalable Transaction Infrastructure: The platform needs highly stable and scalable transaction network.

- Low Transaction Costs: The payment process in any manufacturing transaction currently entails many different steps and middleman to settle a transaction and different fees to pay for payment gateways, thus making transaction fees very high a long route that could be cut short with blockchain technology. Blockchain is the perfect tool for payment to be more efficient and more trustful. Payment processing through blockchain has a significantly bigger potential for transaction speeds and low prices. 
- Chain Management: Companies must manage a complex supply chain of manufacturers, consumer, carriers, and logistics providers. A blockchain of the transaction, containing order details and negotiated fees and commissions (in the form of smart contracts) will be used to capture designs/ products, delivery and possible return events, and drive financial settlement with fewer opportunities for dispute. The nature of blockchain records and native token means that each party can make data visible to the others. All parties have visibility into the transaction and no custom interface must be negotiated between partners (only to/from the blockchain). The blockchain is the system of records for the entire transactions. It reduces time delays, costs, and human errors that plague transactions today, automate inventory control, and streamline fulfillment processes. But, errors to some extent are possible since 3D Printed products are not digital, the blockchain records of their track is inevitably postfactum.

- Designers Network

- Manufacturers Network

- Consumers Network

- Fulfillment Network (operators, workers and couriers) (Figure 11)

Designers publish their design for sale, carry out promotion and loyalty campaigns, and get feedback from consumers. Network enables consumers to order their design or personalized them and facilitates the order for manufacturing process by optimizing the 3D model based on a given specification and material.

Manufactures publish their rate for specific types of materials, level of precision and duration of time to produce objects. Manufacturing network has cross-platform system to distribute

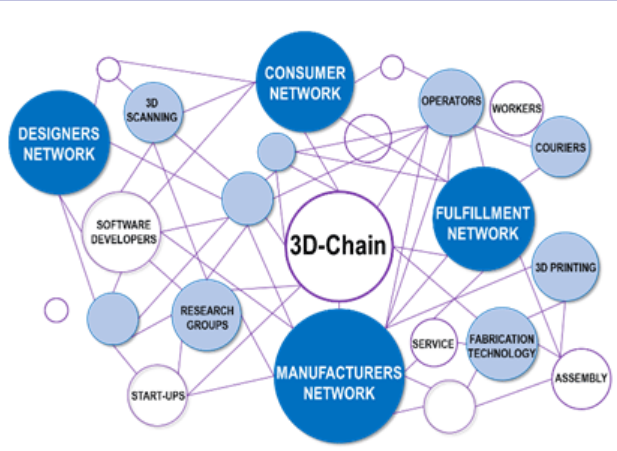

Figure 11: 3D-Chain's network

the design across multiple fabrication technology providers for faster production.

Consumers order a design or product in the network. They also can publish their personalized designs (e.g. celebrities design) or brand design to have more followers, earn credit and ease of the process for other consumers.

All the networks based on Blockchain inherent the security and transparency aspects of the decentralized platforms where designers and manufactures can communicate directly to consumer and have assurance of direct payment with minimum transaction fee. Designers are paid based on every usage of their designs without worrying about copyright enforcement. On the other hand, consumer will get best price with minimum overhead and have confidence that their review and feedback can't be manipulated or deleted. The platform establishes a decentralized, fair and secure platform for execution of an effective incentive model for all parties to join the 3D-Chain ecosystem. Figure 12 shows the whole platform and communications between networks.

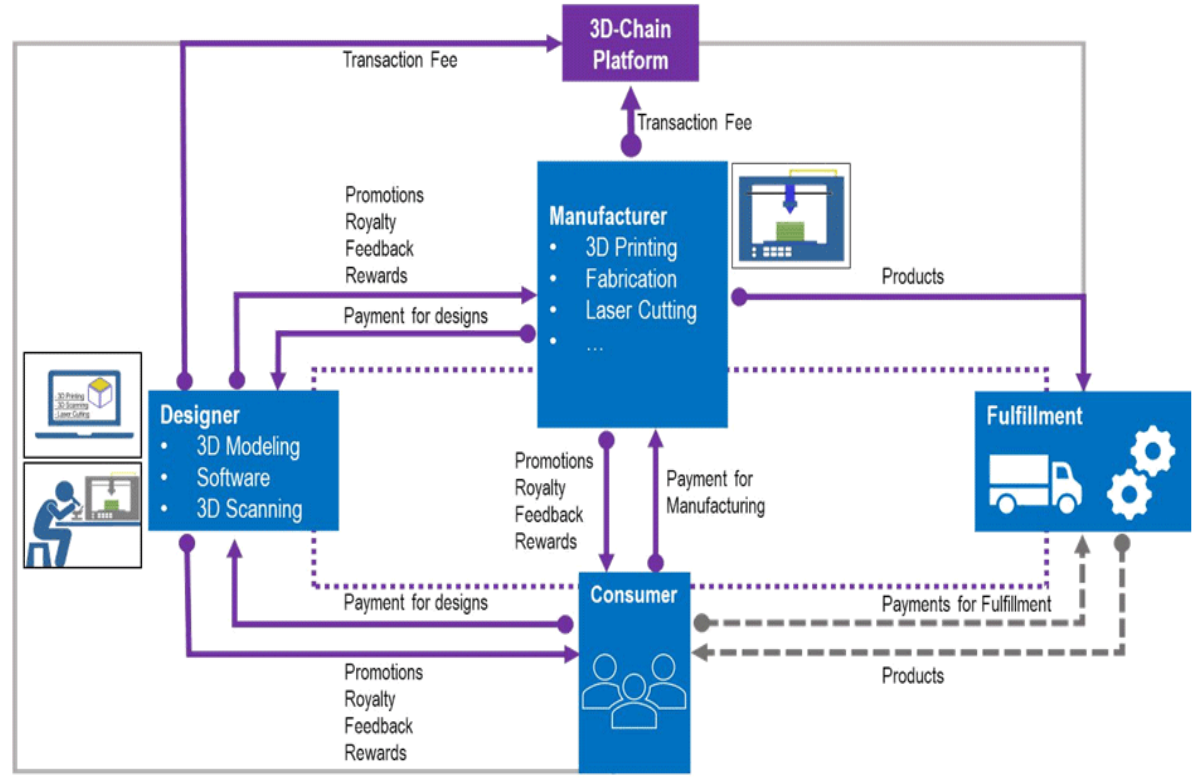

Figure 12: 3D-Chain's network flow 


\section{Designers}

Designers are companies or individuals in the business of designing 3D objects. They can range from individual designers to large designing companies. Designer's roles are:

- Develop designs or personalized a design based on consumers needs

- Make the design compatible with specific 3D Printer and optimize it for the printer

- Work with consumer to select materials

- Provide attractive promotion and loyalty rewards to acquire and retain consumers

\section{Incentive for designers}

- Access to global network

- Secure environment with built-in copyright enforcement

- Get fair prices on their design and compete and collaborate openly with other designers

- Provide compatibility of their design with manufactures

- Serve in a trustful manner to keep a high rating score and attract more consumers

- Get promotion and loyalty rewards from manufacturers

\section{Manufacturers}

Manufacturers are companies or individuals in the business of 3D Printing and fabrication technologies. They could range from individuals at home to large multinationals manufactures. Large or small, no manufacturer has enough power to fulfill exponential growth in consumer's demand. Therefore, consumer can work with as many as manufactures as they want to expedite manufacturing process and keep up with demands. Manufacturer roles include:

- Generate products align with their pre-defined specification and accuracy

- Deliver products ordered by consumers to fulfillment centers

- Work directly with designer and consumer to develop compatible product

\section{Incentive for manufacturers}

- Get fair prices on their technology and compete openly with other manufacturers

- Collaborate with other manufacturers

- Promotion from designers to make their device compatible with their work

- Serve in a trustful manner to keep a high rating score and attract more consumers

- Provide attractive promotion and loyalty rewards to acquire and retain consumers

\section{Consumers}

Consumers are individuals or companies that want to buy a design or make a high-quality product at cheaper prices. Consumer can license an idea or a design to get credit every time other consumer uses their design. Consumer roles include:

- Search for a design listed by designers, make orders for a design or order personalized design and pay for them

- Search for a manufacturer, make orders, and pay for them

- License a design/idea to earn credit and followers

- Participate in feedback requests

- Receive promotion, loyalty, referral and feedback rewards

\section{Incentive for consumer}

- Order customized designs

- Personalized design and access to designers

- Publish personalized designs (e.g. celebrities design) or brand design to have more follower, earn credit/loyalty and ease of the process for other consumer

- Get orders delivered to the doorstep

- Leave feedback to influence manufacturers/designer

- Get different types of rewards

\section{Fulfillment}

Fulfillment can be performed by different centers. Fulfillment center operators are owners or operators of existing warehousing facilities and/or delivery fleet. They provide the space where workers take products delivered by manufacturers and assemble orders. They

- Collect products delivered by manufacturers to fulfillment centers

\section{- Pass orders to couriers}

Courier company or as independent contractors, can also follow instructions received via 3D-Chain fulfillment app to pick up orders from fulfillment centers.

\section{Data processing}

Products in the consumer markets constitute the basic item that actions revolve around. Designers include their designs as a product in the ecosystem and manufacturers rents their devices on the platform with several defined parameters, such as model, material, speed, price, description, etc. that makes it easy to find and choose a 3D Printing or fabrication technology device for ordering.

To choose between designers and manufacturers, a rating and reputation mechanism should be implemented. Also, information on the network is an asset that could be shared between users to take the benefits of the networks. These main aspects of data processing are explained in detail. 


\section{Rating and reputation}

Manufacturers, designers and consumer need some ways to estimate the reputation of others. This is critical for making choices regarding everyday transactions in the network, and it also plays a core role in network governance and resource allocation.

3D-Chain will provide a rating and reputation mechanism to address these needs. Rating system design system will need to evolve along with the network. Ultimately it may equal or exceed the subtle and comprehensive rating systems; but for starters a system will serve adequately as the initial condition for ongoing refinement.

All parties involved in transactions are asked to rate each other after each exchange of services for tokens (or for other services); however, rating is not mandatory.

\section{Data ownership and privacy}

Most of the large companies draw outsized benefits from their ability to build and hoard gigantic datasets, which are then used to extract knowledge from data or even sale them. Outside of these few tech giants, small, innovative companies struggle with access to data, while other large organizations, even when they can gather data, lack the expertise to turn the data into a usable form.

The platform allows network consumer to collaborate in building very large, decentralized datasets, each contributing data in an agreed-upon format. Contributors can then receive payment from any part of the network that uses the data. Data producers can specify privacy restraints and other restrictions on access and use of their data. By decentralizing ownership and access to gigantic datasets, 3D-Chain takes another important step to democratize access to 3D Printing ecosystems and their benefits.

\section{Artificial Intelligence network: A New Value Chain}

With all data available from 3D-Chain's network, 3D-Chain generates a new value chain such as big data and the use of data-gathering sensors (the so-called Internet of Things) for experts in Artificial Intelligence. New machine learning models can be developed to fine-tune products based on customer's demand. Machine learning helps in products and materials recommendation, finding similar products, and optimizing a design for specific fabrication technology. It can also find any flaw in design or manufacturing automatically. Across manufacturing industry, 3D-Chain opens doors for many aspects of machine learning development within the network to increase the performance and generate revenue for participants. These new Artificial Intelligence network can make substantial improvement in manufacturers interaction with consumer and how they manage their operations.

\section{Conclusion}

In this paper we present a new decentralized network to solve current challenges in fourth manufacturing revolution. We present a platform for global growth in manufacturing using 3D printing industry and automated fabrication technologies. By connecting network of manufacturers with different capabilities on a decentralized platform, manufacturers, designers and consumers have the freedom to focus on the application which will cause the real transformation in the industry.

The platform is called 3D-Chain which is based on blockchain technology, proposes a leaner supply chain and fewer risks by creating a digital supply chain for manufacturing. Moreover, 3D-Chain increase collaboration between existing and future players in its decentralized platform. A co-creation framework which integrates manufacturing (hardware, software, service, ...) to drive innovation and serve customers.

\section{References}

1. Zareiyan B, Korjani M. Decentralized Manufacturing: Global Decentralized Network Directly Connecting Manufacturers, Designers, and Consumers. Int J Adv Robot Automn. 2018;3(1):1-5. doi: 10.15226/2473-3032/3/1/00132

2. Stock T, Seliger G. Opportunities of Sustainable Manufacturing in Industry 4.0, Procedia CIRP. 2016;40:536-541. doi: 10.1016/J. PROCIR.2016.01.129

3. Kang HS, Lee JY, Choi S, Kim H, Park JH, Son JY, et al. Smart manufacturing: Past research, present findings, and future directions. International Journal of Precision Engineering and Manufacturing-Green Technology. 2016;3(1):111-128. doi:10.1007/ s40684-016-0015-5

4. Rüßmann M, Lorenz M, Gerbert $\mathrm{P}$, Waldner M, Justus J, Engel $\mathrm{P}$, et al. Industry 4.0: The Future of Productivity and Growth in Manufacturing Industries. BCG. 2015. doi:10.1007/s12599-0140334-4.

5. Hermann M, Pentek T, Otto B. Design Principles for Industrie 4.0 Scenarios. 2016 49th Hawaii International Conference on System Sciences (HICSS). 2016:3928-3937. doi:10.1109/HICSS.2016.488

6. Roblek V, Meško M, Krapež A. A Complex View of Industry 4.0. SAGE Open. 2016. doi:10.1177/2158244016653987

7. Qin J, Liu Y, Grosvenor R. A Categorical Framework of Manufacturing for Industry 4.0 and Beyond, Procedia CIRP. 2016;52:173-178. doi: 10.1016/J.PROCIR.2016.08.005

8. Brettel M, Friederichsen N, Kelle M, Rosenberg M. HowVirtualization, Decentralization and Network Building Change the Manufacturing Landscape: An Industry 4.0 Perspective. International Journal of Information and Communication Engineering. 2014;8(1):37-44.

9. Mueller B. Additive Manufacturing Technologies - Rapid Prototy ping to Direct Digital Manufacturing. Assembly Automation. 2012;32(2). doi: 10.1108/aa.2012.03332baa.010

10.Zareiyan B, Khoshnevis B. Effects of interlocking on interlayer adhesion and strength of structures in 3D printing of concrete. Automation in Construction. 2017;83:212-221. doi:10.1016/j. autcon.2017.08.019

11. Sculpteo. State of 3D Printing 2017. 2017. Available from: https:// www.sculpteo.com/en/get/report/state_of_3D_printing_2017/

12.Stratasys. Trend Forecast: 3D Printing's Imminent Impact on Manufacturing. 2015. Available from: https://www.stratasysdirect. com/content/pdfs/\%0Asys_trend-forecast_v10.pdf 
13. Wohlers TT, Caffrey T. Wohlers Report 2015: 3D Printing and Additive Manufacturing State of the Industry Annual Worldwide Progress Report. Wohlers Associates. 2015.

14. MarketsndMarkets. 3D Printing Market- Global Forecast to 2023. Marketsandmarkets.com. 2017. Available from: https:// www.marketsandmarkets.com/Market-Reports/3d-printingmarket-1276.html

15.Gaub H. Customization of mass-produced parts by combining injection molding and additive manufacturing with Industry 4.0 technologies. Reinforced Plastics. 2016;60(6):401-404. doi: 10.1016/J.REPL.2015.09.004
16.Zawadzki P, Żywicki K. Smart product design and production control for effective mass customization in the industry 4.0 concept. Management and Production Engineering Review. 2016;7(3):105112. doi: 10.1515/mper-2016-0030

17. Zheng P, wang H, Sang Z, Zhong RY, Liu Y, Liu C, et al. Smart manufacturing systems for Industry 4.0: Conceptual framework, scenarios, and future perspectives. Frontiers of Mechanical Engineering. 2018;13(2):137-150. doi: 10.1007/s11465-018-0499-5 